\title{
MISMATCH NEGATIVITY IN PATIENTS WITH SCHIZOPHRENIA
}

\author{
Aleš Urban ${ }^{1,3}$, Jan Kremláček ${ }^{2}$ Jan Libiger ${ }^{1}$ \\ Charles University in Prague, Faculty of Medicine in Hradec Králové and University Hospital Hradec Králové, Czech \\ Republic: Department of Psychiatry ${ }^{1}$, Institute of Pathological Physiology ${ }^{2}$; Outpatient Department of Psychiatry, Brandýs \\ nad Labem, Czech Republic ${ }^{3}$
}

Summary: Cognitive deficit is considered to be a part of core dysfuncions in schizophrenia. It is associated with social impairment and influences the long-term course of the disorder. In addition to neuropsychological methods, event-related potentials can be used to study cognitive functions. In patients with schizophrenia an association was found between amplitude changes in slow negative component of evoked responses and infrequent deviations in a series of uniform stimuli. This amplitude change is known as "mismatch negativity“ (MMN). It is supposed to be independent of the focused attention and effort that otherwise interfere with neuropsychological testing. Recently accumulated knowledge on MMN as a possible preattentive measure of cognition supports its potential significance for neuropsychological assessment. It may be helpful in more precise diagnosis and functional evaluation of schizophrenia.

Keywords: Schizophrenia; Event related potentials; Mismatch negativity; Cognitive functions; Sensory memory; Working memory; $N$-methyl-D-aspartate receptors.

\section{Introduction}

Schizophrenia is a serious mental condition affecting 0.5 to $1 \%$ of population. As a group, patients with schizophrenic disorder have increased mortality and morbidity. They also have decreased social competency. Both characteristics are associated with high socioeconomic costs. Schizophrenia is characterized by a number of symptoms that are usually divided into two groups: the positive symptoms include hallucinations, structural and content-related thought disturbances, behavioral disturbances, and negative symptoms include blunt affect/emotionality, impoverished speech, volitional disturbances, and anhedonia. The disorder is also associated with deficit in a number of cognitive areas, particularly attention, memory, and executive functions. Cognitive deficit is an essential part of schizophrenic disorder. It may be thought of as a defect that is the hidden, i.e. primary "core" giving rise to the whole of subsequently developing psychopathology of schizophrenia. Symptoms of cognitive impairment have been found to be significant predictors of the severity of the course of the schizophrenic disorders and also the degree of the patient's social impairment.

The basis of cognitive dysfunction has not yet been fully clarified. Structural theories suppose the presence of a deficit located predominantly in prefrontal and/or temporal and hippocampal areas. Recently, however, new evidence has emerged, suggesting that the damage, both structural and functional, is more extensive and involves more areas across the brain. Neurochemical theories associate schizophrenia with disturbances in the systems of dopaminergic and N-methyl-D-aspartate (NMDA) glutamatergic neurotransmission (19).

Cognitive dysfunction in patients with schizophrenia can be investigated also by electrophysiological studies of the cognitive evoked potentials. Event related potentials (ERPs) are recorded analogously to an electroencephalogram (EEG) as a response of the central nervous system (CNS) to sensory stimuli.

The subject is exposed to a series of stimulation impulses. The final ERP is an average of EEG recorded after separate stimuli. The waveform of the resulting response depends on stimulation parameters and the cognitive task performed by the examined subject. It can be described as an arrangement of several monophasic waves of various polarity. Individual waves are characterized by amplitude and latency of their maxima or minima. Latency describes the time lag of a wave related to the stimulus administration. Recording from multiple electrodes make possible to study the topography of these parameters on the scalp.

One of the most robust electrophysiological findings in patients with schizophrenia established with ERPs is the deficit in the generation of the P300 wave. This deficit has been first described more than 25 years ago (36), and has subsequently been confirmed by a number of studies. In an effort to locate areas that participate in the generation of 
the P300 wave, intracerebral electrodes were used with surprising result. The stimulation activated areas of the brain that are not essential to processing the stimulus. The generation of the P300 wave can involve up to 9 various cerebral areas (13). This finding points out the problem associated with the interpretation of the source of deviations in ERPs (14).

Over the last ten years, the attention of investigators in patients with schizophrenia has shifted to cognitive components of evoked potentials that precede the P300 wave and reflect earlier stages of information processing (17). The objective has been found disturbances in information processing that are involved in cognitive dysfunctions of patients with schizophrenia. Detection and interpretation of the disturbance results in better understanding of the pathophysiology of schizophrenia. It may help to better assess the dysfunction in individual patients, and eventually bring new therapeutic approaches. One of the components of evoked potentials that brought significant insights is "mismatch negativity“ (MMN).

\section{What is ,mismatch negativity“ (MMN)?}

„Mismatch negativity“ is defined as the negative ERP component that reflects processes associated with automatic detection of change. MMN was first described by Näätänen and co-workers for acoustic stimuli in 1978 (30). MMN reflects neuronal disagreement between the representations of current (deviant) stimulus and previous (standard) stimuli (29). MMN follows, with a latency of 200 to $400 \mathrm{~ms}$ after stimulus presentation. It comes after the sensory component, and precedes the possible cognitive component of evoked potentials (see Fig. 1). MMN represents a higher-level of information processing compared to the primary sensory detection at the cortical level (20).

A deviant stimulus for MMN in the auditory modality can differ from the standard stimulus in terms of duration, frequency, intensity, spatial location, phoneme variation, etc. (28).

In order to elicit the MMN, a memory trace with the characteristics of standard stimulus must be present. It is

\section{Stimulation diagram for MMN}

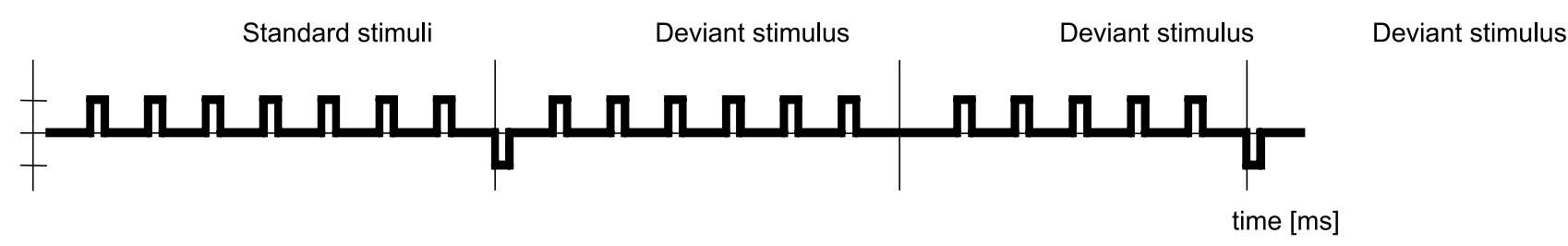

\section{MMN response}

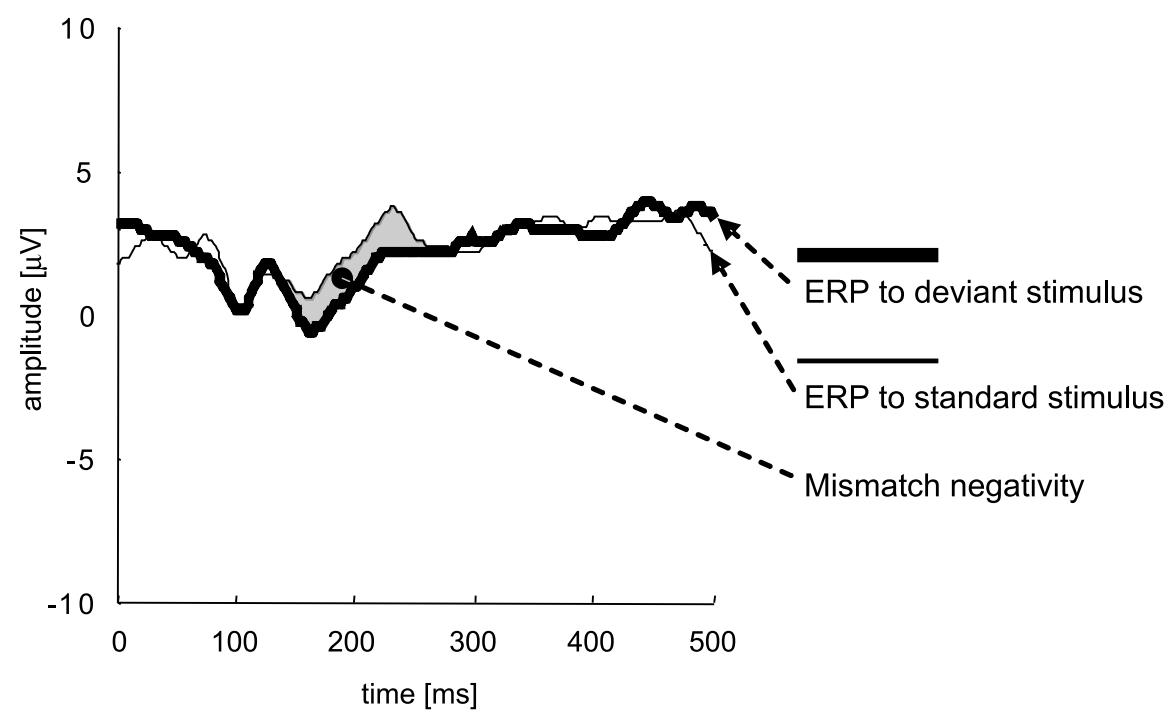

Fig. 1: Diagram shows how stimulation produces "mismatch negativity". Note that it is possible to present deviant stimulus in the form of a missing standard stimulus. The lower section of the figure shows a response obtained through averaging 40 individual responses to standard stimuli (thin line) and similarly produced ERP as a response to the deviant stimulus (full line). The so-called "mismatch negativity" is represented by a gray area between the lines. 
necessary to present repeated standard stimuli (23). Presentation of a single stimulus is not sufficient to generate MMN.

MMN latency is the function of time necessary to differentiate a deviant from the standard stimulus. Increasing the interstimulus interval can result in extending the MMN latency. The memory trace of standard stimulus is probably less accurate with longer intervals, and this makes the detection of a deviant stimulus more difficult (39).

The extension of the interval between stimuli is associated with decreasing MMN amplitude, probably because of a weaker memory trace (8). MMN is not elicited by a stimulus change when the memory trace has already disappeared. MMN amplitude is generally known to increase in proportion to the difference between standard and deviant stimuli (35). It also increases with the number of standard stimulus repetitions (38).

Acoustic MMN is supposed to be generated in the neurons of the temporal cortex which respond specifically to stimulus change. Neurons of the frontal cortex probably also participate during the generation (35). Major generators of MMN were identified in the supratemporal auditory cortex area by the distribution of MMN over the scalp, magnetoencephalography studies, intracranial sensing of electrical activity and cerebral lesions. The precise location of these generators probably depends on stimulus characteristics. The role of the frontal cortex in the generation of MMN is supported by the finding of missing MMN in patients with dorsolateral prefrontal cortex area defects (1).

Most studies of MMN done so far have been concerned with auditory stimuli. There is some evidence of the existence of MMN in other sensory modalities. In spite of the inconsistency of these findings, there seems to exist an analogy to auditory MMN in somatosensory (22), visual $(25,33,43)$ and olfactory modalities $(24)$. However, the assumption of the existence of a common processing mechanism for various information raises questions as to why it is so difficult to detect MMN in those other modalities (35)? One possible answer can be in the different role for attention in various sensory inputs. Another possible explanation is different complexity of sensory analysis (particularly in the visual modality) required for the MMN eliciting. The objective, non-invasive nature of the recording techniques used for MMN and its low cost seem to hold promise for its potential use in clinical research $(28,27)$.

\section{MMN and Psychophysiology}

The psychophysiological processes underlying to MMN have not been clarified as of yet. Näätänen and Winkler propose the existence of two distinct phases of auditory information processing. The first phase is concerned with the analysis of particular sound properties. They are combined into auditory perceptions or representations in the second phase. Both authors assume the auditory N1 to reflect the first phase, while the MMN corresponds to the second phase (29).
The original work on MMN assumed that MMN occurs automatically, regardless of whether the subject pays attention to presented deviant stimuli or not (30). It was possible to elicit MMN even when auditory stimuli were masked with visual stimuli (1). MMN recorded in comatose patients also supports its independence of attention (9). However, other studies suggest the possibility that attention can influence MMN. Differences in terms of amplitude among individual groups of patients can also be interpreted as different strategies in attending to sensory stimulation. Patients with schizophrenia also have difficulties with sustained attention.

\section{Relationship of MMN to working memory}

The neuropsychological concept of working memory describes the ability of the brain to code, retain and manipulate information over brief periods of time (3). It consists of two main components: central executive component of prefrontal cortex and modality-specific transitory auxiliary systems (41), that serve a transitory information storage. These systems include phonological system (phonological loop) in auditory modality and visuo-spatial system (visualspatial sketchbook) in visual modality (3). The transient auxiliary system directly performs operations, while the central executive functions are responsible for selection, steering and control the auxiliary systems (12).

A number of researchers regard $\mathrm{MMN}$ as the indicator of a well functioning auditory sensory memory (40). Auditory sensory memory, the most elementary component of working memory, works independently of attention. MMN is known to occur when a deviant stimulus differs from the standard stimulus. Because presentation of a single stimulus is not sufficient to produce MMN, it seems obvious that processing after the deviant stimulus occurs concurrently with the comparison of a current stimulus with the memory trace of a standard stimulus. This leads to the conclusion that MMN can be used as an objective indicator of sensory memory. This was indirectly confirmed in a study with healthy volunteers in which auditory memory trace and MMN lasted similar periods of time and had similar sensitivity to masking with different stimuli (48).

Because a working memory deficit is present in patients with schizophrenia, MMN can be used for its measurement.

\section{Does MMN reflect a dysfunction of the glutamatergic receptor system?}

Activation of NMDA receptors plays a key role in learning and initiating long-term memory formation. NMDA receptors are also important in working memory (19). The density of NMDA in the neocortex and hippocampus is similar. Javitt employed intracortical sensing of electrical activity in the anterior insula concurrently with focal infusion of NMDA- and non-NMDA-antagonists into the micro-re- 
gion of a sensing electrode. His study has shown that locally administered NMDA antagonists prevent the occurence of MMN, while it has no effect on any other components of auditory evoked potential $(19,46)$.

\section{MMN in Schizophrenia}

Insufficient formation of MMN in patients with schizophrenia was first described in the early nineties (41). More than sixty studies have been published on the relationship between MMN and schizophrenia since then. For a meta-analysis see Umbricht nad Krljes (44). Most of these studies found that both untreated and treated patients with schizophrenic disorder had lower MMN amplitudes than healthy controls. Only a small number of these studies found no MMN generation deficit (31). These findings are based on the assumption of a neurophysiological disturbance at the level of the auditory sensory cortex in schizophrenia, corresponding damaged auditory memory and anatomical abnormalities in the superior temporal gyrus $(15,18)$. Disturbed auditory sensory memory of patients with schizophrenia is revealed in a decreased accuracy of retrieval of the corresponding memory trace. Similar findings have been described for the visual sensory memory system (10).

One influential hypothesis of the pathogenesis of schizophrenia regards the disturbance of NMDA receptors in the glutamatergic system as the key neurochemical dysfunction. This hypothesis is supported by observations of the effects produced by phencyclidine, ketamine and other NMDA glutamatergic receptor antagonists in healthy volunteers. These substances induce symptoms that resemble symptoms of patients with schizophrenia (19). Administration of these substances can also reduce MMN, or even lead to its extinction. The change in MMN generation in patients with schizophrenia can be linked to disturbances in glutamatergic neurotransmission in NMDA receptors in the area of the auditory sensory cortex (16). To confirm the role of the NMDA receptors in working memory Umbricht conducted a study with healthy volunteers who received ketamine. The deficit in the working memory of patients with schizophrenia was similar to the deficit in healthy controls after ketamine administration (46). The process of acquiring and coding information was more affected than that of retaining information.

Another supportive finding is the impairment of MMN generation induced with acetylcholinesterase inhibitor tetrahydrominoacridine in patients with Alzheimer's disease (2). Lower MMN following the use of cholinomimetic was explained by an increased cholinergic modulation of NMDA receptors in the auditory cortex.

The relationship between deteriorated cognitive function and MMN was studied by Baldeweg. He found a negative correlation between MMN and the degree of neuropsychological impairment (4). However, there are other findings that do not support the association of MMN, working memory, and NMDA receptor dysfunction. A double blind placebo-controlled study with healthy volunteers failed to confirm expected changes in MMN after the administration of ketamine (32).

Baldeweg reports a greater MMN deficit in the area of the front central electrode. This is consistent with the preferential damage to frontal MMN generators. According to the author, this finding is specific for schizophrenia $(4,5)$. Other similar findings suggest that patients with schizophrenia differ in their MMN topography from those with other neuropsychiatric disorders.

The generation of MMN is indirectly proportional to the prevalence of negative symptoms of schizophrenia. However, it is independent of a patient's current schizophrenia psychopathology (16).

Umbricht's study found no difference in MMN among patients with one episode compared to those with repeated episodes (45). A study in first episode patients did not find MMN deficit (37). On the other hand, another study detected an increase in the MMN deficit with disease progression (42). Inconsistent findings come also from healthy first-degree relatives. A study done by Jessen found lower amplitude in relatives of patients with schizophrenia (21). This was not confirmed in another recent study (6). Brockhaus-Dumke found suprisingly higher MMN amplitude in a subgroup of untreated first episode patients (7). These findings support the assumption that MMN amplitude changes during the course of schizophrenia.

This latter study also examined a group of patients with prodromal symptoms. The results were variable and heterogeneous (7).

Other authors regard the MMN generation deficit as a vulnerability marker of schizophrenic disorder ("trait marker"). Difference in MMN between schizophrenia and other neuropsychiatric disorders supports this interpretation. Umbricht assumes that MMN can be an endophenotype (an important hidden hereditary characteristic) of a subgroup of patients with schizophrenia with abnormal glutamatergic neurotransmission due to NMDA receptor dysfunction (45). Contrary to that, Bramon regards the MMN deficit as a nonspecific vulnerability marker that cannot serve as an endophenotype of schizophrenia (6).

Treatment with antipsychotics affects P300. MMN has not changed after treatment with clozapine (47). MMN deficit is also not influenced by treatment with conventional or atypical antipsychotics (34). No difference was found in the amplitude of those treated in comparison to untreated patients with schizophrenia. The dose of antipsychotic or anticholinergic medication has not been found to correlate with the amplitude (20). Also, no change was found when patients were switched from classical to new antipsychotics (47).

A recent study carried out according to stringent methodology found an association of the deficit in MMN generation with worse social functioning. This deficit can be probably used as a predictor of functional deterioration in patients with schizophrenia (26). 


\section{Conclusion}

MMN is a significant electrophysiological measure of cognitive deficit. It reflects deficits in information processing at primary and secondary sensory cortex levels (45). Investigation of $\mathrm{MMN}$ is non-invasive, inexpensive, and can be done quickly. Its reproducibility is comparable with that of other, broadly used neuropsychological tests $(26,27)$. Compared to other investigations, it probably depends less on variable attention level, motivation and effort of the patient (20). The issue of attention, however, deserves clarification.

MNN is commonly regarded to be an index of the sensory memory. An alternative view takes into account the role of the frontal generator and suggests that it can also be related to the executive component of working memory. Further inquiry into MMN can contribute to the identification of a subgroup of patients who exhibit dysfunction of NMDA glutamatergic neurotransmission and also have worse cognitive and social functioning.

Whether MMN generation reflects the information processing and implicit memory or is rather a disturbance of working memory or executive functions is unresolved. Patients with schizophrenia have also been described as having deficit in the generation of the early negative peak of evoked potential preceding MMN - so-called N1 (11). This finding supports the hypothesis that schizophrenia is characterized by a disturbance in information processing at very early stages. Evidence of disturbed information processing can be found at all levels - the primary cortex, sensory memory, and executive component of the working memory. It is quite possible that a deficit in MMN generation reflects a disturbance of the functional interconnections between various brain areas.

The study of the deficit in the genesis of MMN in patients with schizophrenia is a new approach to pathophysiological mechanisms in this disorder. The question of whether this deficit is common to various sensory modalities or specific for a particular sensory system is open to inquiry. It may have considerable importance for understanding the nature of schizophrenia.

The work on this article was supported by the Czech Ministry of Education Research Objective Grant MSM 0021620816.

\section{References}

1. Alho K, Woods DL, Algazi A. Lesions of frontal cortex diminish the auditory mismatch negativity. Electroencephalogr Clin Neurophysiol 1994;91:353-362.

2. Aramakis VB, Bandrowski AE, Ashe JH. Activation of muscarinic receptors modulates NMDA receptor-mediated responses in auditory cortex. Exp Brain Res 1997;113:484-496.

3. Baddeley A. Working memory. Science 1992;255:556-559.

4. Baldeweg T, Klugman A, Gruzelier J, Hirsch SR. Mismatch negativity potentials and cognitive impairment in schizophrenia. Schizophr Res 2004;69:203-217.

5. Baldeweg T, Klugman A, Gruzelier J, Hirsch SR. Impairment in frontal but not temporal components of mismatch negativity in schizophrenia. Int $\mathrm{J}$ Psychiphysiol 2002;43:111-122.
6. Bramon E, Croft RJ, McDonald C, Virdi GK, Gruzelier JG, Baldeweg T, Sham PC, Frangou S, Murray RM. Mismatch negativity in schizophrenia: a family study. Schizophr Res 2004;67(1):1-10.

7. Brockhaus-Dumke A, Tendolkar I, Pukrop R, Schultze-Lutter F, Klosterkotter J, Ruhrmann S. Impaired mismatch negativity generation in prodromal subjects and patients with schizophrenia. Schizophr Res 2005 Mar 1;73(2-3):297-310.

8. Cowan N, Winkler I, Wolgang T, Näätänen R. Memory prerequisites of mismatch negativity in the auditory event-related potential (ERP). J Exp Psychol Learn Mem Cogn 1993;52:559-563.

9. Fischer C, Morlet D, Giard M-H. Mismatch negativity and N 100 in comatose patients. Audiol Neurootol 2000;5:192-197.

10. Fleming K, Goldberg TE, Binks S, Randolph Ch, Gold JM, Weinberger DM. Visuospatial working memory in patients with schizophrenia. Biol. Psychiatry 1997;41:43-49.

11. Ford JM, Mathalon DH, Kalba S, Marsh L, Pfefferbaum A. N1 and P300 abnormalities in patients with schizophrenia, epilepsy, and epilepsy with schizophrenialike features. Biol Psychiatry2001;49(10):848-60.

12. Goldman-Rakic PS. Working memory dysfunction in schizophrenia, J Neuropsychiatr Clin Neurosci 1994;6:348-357.

13. Halgren E, Marikovic K, Chauvel P. Generators of late cognitive potentials in auditory and visual odball tasks. Electroencephalogr Clin Neurophysiol 1998;106: 156- 164 .

14. Hari R, Joutsiniemi SL, Sarvas J. Spatial resolution of neuromagnetic records theoretical calculations in a spherical model. Electroencephalogr Clin Neurophysiol 1988;71:64-72.

15. Holcomb HH, Ritzl EK, Medoff DR, Nevitt J, Gordon B, Tamminga CA. Tone discrimination performance in schizophrenic patients and normal volunteers: impact of stimulus presentation levels and frequency differences. Psychiatry Res. 1995;57:75-82

16. Javitt DC, Shelley AM, Silipo G, Lieberman JA. Deficits in Auditory and Visual Context-Dependent Processing in Schizophrenia. Arch Gen Psychiatry 2000; 97:1131-1137.

17. Javitt DC, Grochowski S, Shelley AM, Ritter W. Impaired mismatch negativity (MMN) generation in schizophrenia as a function of stimulus deviance, probability, and interstimulus interval. Electroencephalography and clinical Neurophysiology 1998;108:143-453.

18. Javitt DC, Strous RD, Grochowski S, Ritter W, Cowan N. Impaired precision, but normal retention, of auditory sensory („echoic“) memory information in schizophrenia. J Abnorm Psychol 1997;106:315-324.

19. Javitt DC, Steinschneider M, Schroeder CE, Arezzo JC. Role of cortical N-methyl-D-aspartate receptors in auditory sensory memory and mismatch negativity generation: Implications for schizophrenia. Proc Natl Acad Sci USA 1996;93: $11962-11967$.

20. Javitt DC, Doneshka P, Grochowski S, Ritter W. Impaired mismatch negativity generation reflects widespread dysfunction of working memory in schizophrenia. Arch Gen Psychiatry 1995;52:550-558.

21. Jessen F, Fries T, Kucharski C, Nishimura T, Hoenig K, Maier W, Falkai P, Heun $\mathrm{R}$. Amplitude reduction of the mismatch negativity in first-degree relatives of patients with schizophrenia. Neurosci Lett. 2001;309(3):185-188.

22. Kekoni J, Hämäläinen H, Saarinen M, Gröhn J, Reinikainen K, Lehtokoski A, Näätänen R. Rate effect and mismatch responses in the somatosensory system: ERP recordings in humans. Biol Psychol 1997;46:125-142.

23. Kirino E, Inoue R. The relationship of mismatch negativity to quantitative EEG and morphological findings in schizophrenia. Journal of Psychiatric Research 1999;33:445-456

24. Krauel K, Schott P, Sojka B, Pause BM, Ferstl R. Is there a mismatch negativity analogue in the olfactory event-related potentials? Psychophysiol 1999;13:49-55.

25. Kremláček J, Kubová Z, Chlubnová J, Kuba M. Motion-onset VEPs in mismatch negativity paradigm. Perception 2001; S30:62.

26. Light GA, Braff DL. Mismatch negativity deficits are associated with poor functioning in schizophrenia patients. Arch Gen Psychiatry 2005;62:127-36.

27. Näätänen R. Mismatch Negativity: Clinical research an possible applications. International Journal of Psychophysiology 2003;48:179-188.

28. Näätänen R, Escera C. Mismatch Negativity: Clinical and Other Applications. Audiol Neurootol 2000;5:105-110.

29. Näätänen R, Winkler I. The concept of auditory stimulus representation in cognitive neuroscience. Psychol Bull 1999;125:826-859.

30. Näätänen R, Gaillard AWK, Mäntysalo S. Early selective-attention effect reinterpreted. Acta Psychologica 1978;42:313-329.

31. O’Donnell BF, Hokama H, McCarley RW, Smith RS, Salisbury DF, Mondrow E, Nestor PG, Shenton ME. Auditory ERPs to non-target stimuli in schizophrenia: relationship to probability, task demands, and target ERPs. Int J Psychophysiol 1994;17:219-231.

32. Oranje B, van Berkel BNM, Kemnre C, van Ree JM, Kahn RS, Verbaten MN. The Effects of a Sub-Anaesthetic Dose of Ketamine on Human Selective Attention. Neuropsychopharmacology 2000;22:293-302. 
33. Pazo-Alvarez P, Cadaveira F, Amenedo E. MMN in the visual modality: a review. Biol Psychol 2003;63:199-236.

34. Pekkonen E, Hirvonen J, Ahveninen J, Kahkonen S, Kaakkola S, Huttunen J, Jaaskelainen IP. „Memory-based comparison process not attenuated by haloperidol: a combined MEG and EEG study." Neuroreport 2002;13:177-181.

35. Picton TW, Alain C, Otten L, Ritter W, Achim A. Mismatch Negativity: Different Water in the Same River. Audiol Neurootol 2000;5:111-139.

36. Pritchard WS. Cognitive event-related potential correlates of schizophrenia, Psychol Bull 1986;100:43-66.

37. Salisbury DF, Shenton ME, Griggs CB, Bonner-Jackson A, McCarley RW. Mismatch negativity in chronic schizophrenia and first-eposide schizophrenia. Arch Gen Psychiatry 2002;59:686-694.

38. Sams M, Hari R, Rif J, Knuutila J. The human auditory sensory memory trace persists about $10 \mathrm{msec}$ : Neuromagnetic evidence. J Cogn Neurosci 1993;5:363-370

39. Schröger E, Winkler I. Presentation rate and magnitude of stimulus deviance effects on human pre-attentive change detection. Neurosci Lett 1995;193(3): 85-188.

40. Shelley AM, Silipo G, Javitt DC. Diminished responsiveness of ERPs in schizophrenic subjects to changes in auditory stimulation parameters: implications for theories of cortical dysfunction, Schizophrenia Research 1999;37:65-79.
41. Shelley AM, Ward PB, Catts SV, Michie PT, Andrews S, McConaghy N. Mismatch negativity: An index of a preattentive processing deficit in schizophrenia. Biol Psychiatry 1991;30:1059-1062.

42. Shinozaki N, Yabe H, Sato Y. The difference in Mismatch negativity between the acute and post-acute phase of schizophrenia. Biol Psychol 2002;59:115-119.

43. Tales A, Newton P, Troscianko T, Butler S. Mismatch negativity in the visual modality. NeuroReport 1999;10:3363-3367.

44. Umbricht D, Krljes S. Mismatch negativity in schizophrenia: a meta-analysis. Schizophrenia Research 2005;76:1-23.

45. Umbricht D, Koller R, Schmid L, Skrabo A, Grübel C, Huber T, Stassen H. How Specific Are Deficits in Mismatch Negativity Generation to Schizophrenia. Biol Psychiatry 2003;53:1120-1131.

46. Umbricht D, Vollenweider FX, Schmid L, Koller R. NMDA and 5-HT2A receptor dysfunction in working memory deficits in schizophrenia. Biol Psychiatry 2000;47:53S.

47. Umbricht D, Javitt D, Novak G, Bates J, Pollack S, Lieberman J, Kane J. Effects of Clozapine treatment on Auditory Event-Related Potentials in Schizophrenia. Biol Psychiatry 1998;44:716-725.

48. Winkler I, Näätänen R. Event-related potentials in auditory backwards recognition masking: a new way to study the neurophysiological basis of sensory memory in humans, Neurosci. Lett. 1992;140:239-242.

Submitted December 2006.

Accepted January 2007.

\section{Corresponding author:}

Aleš Urban, M.D., Dipl. Ing.,

Z. Fibicha 1460, 25002 Stará Boleslav, Czech Republic, e-mail: a.urban@centrum.cz 